\title{
A large perforation in the piriform sinus during Zenker diverticulotomy effectively closed with "clips-and-rubber band" technique
}

Endoscopic treatment of Zenker's diverticulum by diverticulotomy consists of cutting the septum between the esophageal lumen and the diverticulum, thus restoring bolus transit. This technique is being increasingly preferred to surgical treatment as it is safe and minimizes the in-hospital stay, especially in patients with important co-morbidities [1-2]. Nevertheless, endoscopic diverticulotomy carries a risk of perforation and delayed bleeding [1].

We report here the case of a 94-year-old woman referred for endoscopic treatment of a Zenker's diverticulum causing dysphagia for solids and liquids. We first introduced the diverticuloscope (Cook Medical, Limerick, Ireland) but the progression was difficult and the patient experienced a cough during introduction. We removed the diverticuloscope and noticed a large transmural perforation of the piriform sinus ( $\mathbf{F i g . 1}$ a, $\triangleright$ Fig. 2 a). However, before closing the perforation, we decided to perform the diverticulotomy, without the diverticuloscope, using the window technique [2].

After this, we tried to close the perforation but usual clipping appeared impossible, as it was difficult to catch the two edges of the defect. Therefore, as previously described for closure of the resected area after endoscopic mucosal resection [3], we placed the first clip with a rubber band attached on the lower edge of the perforation by folding the nonperforated mucosa ( Fig.1 b, Fig.2b). Then, a second clip grasped the rubber band and was attached on the upper edge of the perforation ( $\mathbf{F i g} \mathbf{1} \mathbf{c}$, $>$ Fig. 2c). Owing to the elastic force, the margins of the defect were stretched and approached each other, and the entire defect was closed with two additional clips ( $>$ Fig. 1 d, > Fig. 2 d, \ Video 1).

After 3 days of a liquid diet, the patient resumed normal feeding and was discharged on Day 7. At 1 month follow-up

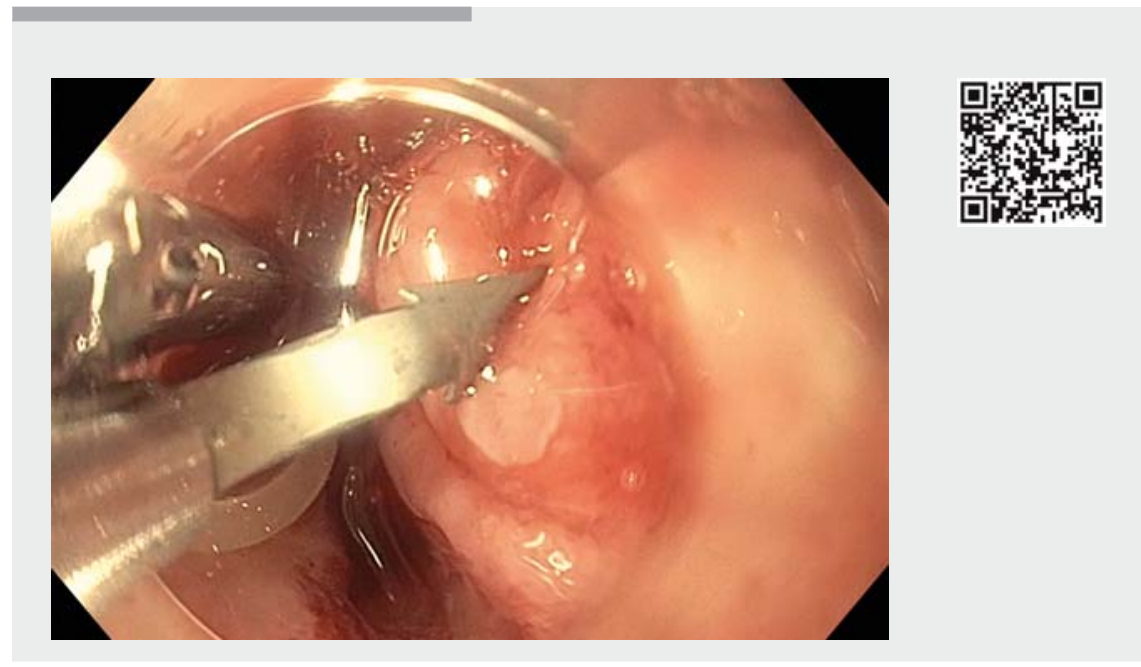

$\checkmark$ Video 1 Effective closure of a large perforation of the piriform sinus using the "clipsand-rubber band technique."
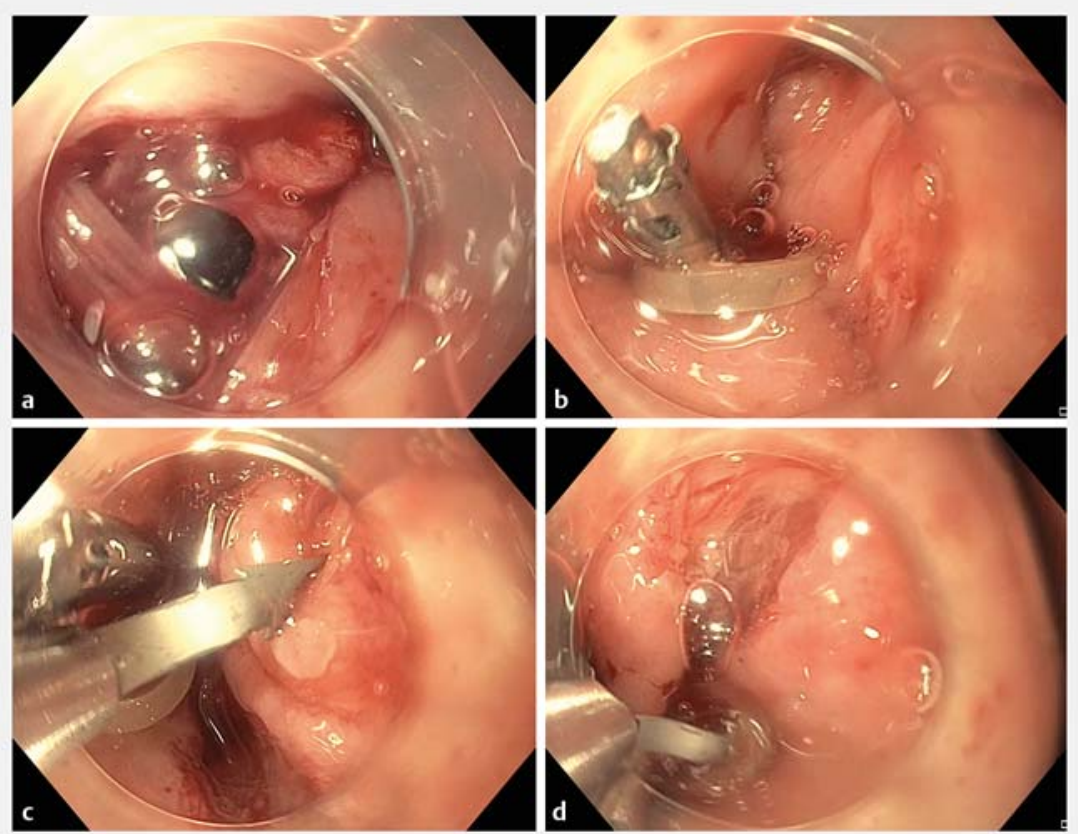

- Fig. 1 Endoscopic view of the "clips-and-rubber band" technique to close a large perforation of the piriform sinus following insertion of a diverticuloscope during endoscopic diverticulotomy. a Large perforation of the piriform sinus. $\mathbf{b}$ Positioning of the first clip with rubber band attached on the lower edge of the defect. c Positioning of the second clip, with grasping and traction of the rubber band. $\mathbf{d}$ Positioning of subsequent clip, with closure of the defect. 


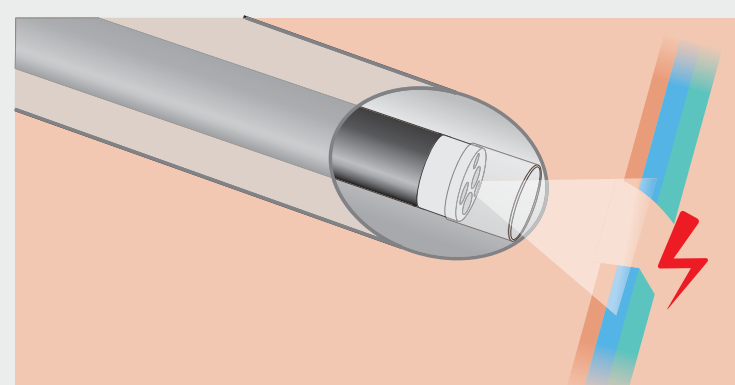

a

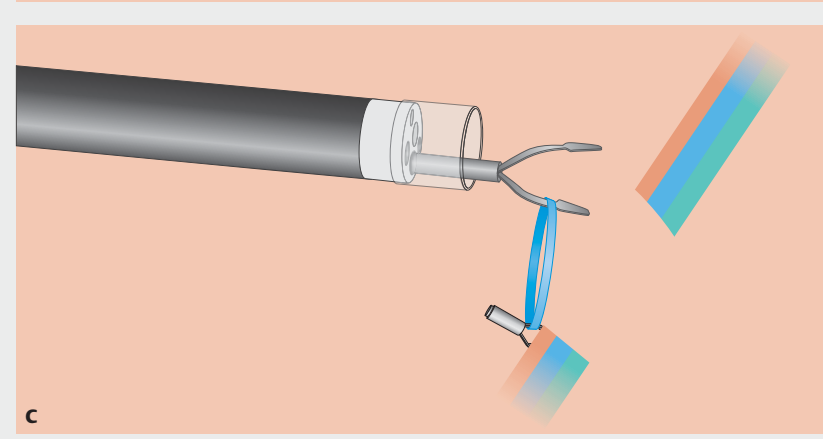

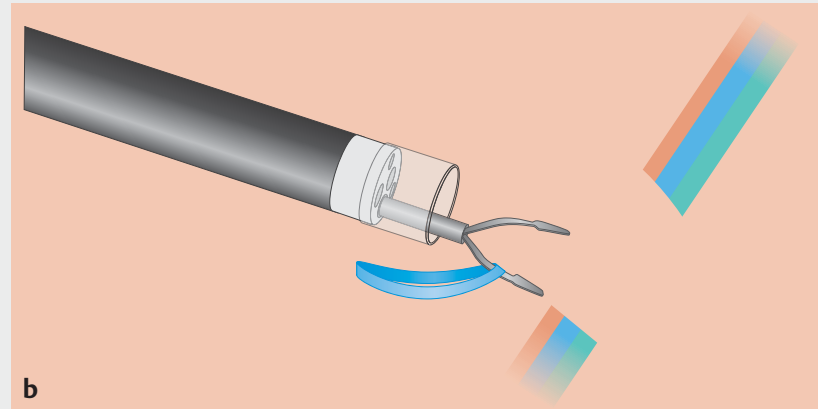

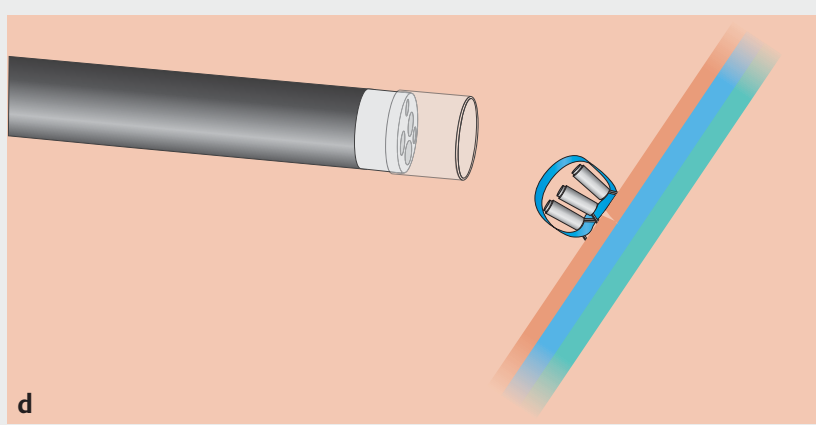

- Fig. 2 Schematic of the "clips-and-rubber band" technique. a Large perforation of the piriform sinus. b The clip with anchored rubber band is positioned on the lateral edge of the defect, folding the mucosa. $\mathbf{c}$ The subsequent clip grasps the rubber band, pulling it across the defect; the clip is applied on the opposite margin of the defect. $\mathbf{d}$ Complete closure of the defect.

after the operation, she was asymptomatic for dysphagia and no sepsis had occurred.

In conclusion, the "clips-and-rubber band" technique allows the close approximation of the edges of large perforations, including those in difficult locations such as the piriform sinus, thus facilitating complete closure.

\section{Endoscopy_UCTN_Code_CPL_1AH_2AJ}

\section{Acknowledgment}

Dr. Leonardo Frazzoni received a travel grant from the European Society of Gastrointestinal Endoscopy to join our team in Edouard Herriot Hospital for 1 month.

\section{Competing interests}

None
The authors

Leonardo Frazzoni ${ }^{1}$, Jérôme Rivory ${ }^{2}$, Laura

Calavas $^{2}$, Jérémie Jacques ${ }^{3}$, Florian Rostain ${ }^{2}$,

Thierry Ponchon ${ }^{2,4}$, Mathieu Pioche ${ }^{2,4}$

1 Department of Medical and Surgical Sciences, Sant'Orsola-Malpighi University Hospital, Bologna, Italy

2 Department of Endoscopy and Gastroenterology, Pavillon L, Edouard Herriot Hospital, Lyon, France

3 Dupuytren University Hospital, Limoges, France

4 Inserm U1032 LabTau, Lyon, France

\section{Corresponding author}

\section{Mathieu Pioche, MD}

Endoscopy Unit - Digestive Disease Department, Pavillon L - Edouard Herriot Hospital, 69437 Lyon Cedex, France Fax: +33-4-72110147 mathieu.pioche@chu-lyon.fr

\section{References}

[1] Ishaq S, Hassan C, Antonello A et al. Flexible endoscopic treatment for Zenker's diverticulum: a systematic review and meta-analysis. Gastrointest Endosc 2016; 83: 1076 1089

[2] Rivory J, Almahayawi A, Roman S et al. Endoscopic Zenker diverticulotomy using the window technique: a technical trick to improve the field of view. Endoscopy 2016; 48: E63-E64

[3] Lupu A, Jacques J, Rivory J et al. Closure of a mucosal defect with clips and rubber band: a technical trick to improve edge apposition in large mucosal defects. Endoscopy 2018; 50: $726-727$

\section{Bibliography}

DOI https://doi.org/10.1055/a-0826-4179

Published online: 7.2.2019

Endoscopy 2019; 51: E79-E80

(c) Georg Thieme Verlag KG

Stuttgart · New York

ISSN 0013-726X 